\title{
Phytotherapy for benign prostatic hyperplasia
}

\author{
Timothy J Wilt*, Areef Ishani, Indulis Rutks and Roderick MacDonald \\ Minneapolis VA Center for Chronic Diseases Outcomes Research, 1 Veterans Drive (1 11 1-0), Minneapolis, \\ MN 55417, USA
}

\begin{abstract}
Objective: To systematically review the existing evidence regarding the efficacy and safety of phytotherapeutic compounds used to treat men with symptomatic benign prostatic hyperplasia (BPH).

Design: Randomized trials were identified searching MEDLINE (1966-1997), EMBASE, Phytodok, the Cochrane Library, bibliographies of identified trials and review articles, and contact with relevant authors and drug companies. The studies were included if men had symptomatic benign prostatic hyperplasia, the intervention was a phytotherapeutic preparation alone or combined, a control group received placebo or other pharmacologic therapies for BPH, and the treatment duration was at least 30 days. Key data were extracted independently by two investigators.

Results: A total of 44 studies of six phytotherapeutic agents (Serenoa repens, Hypoxis rooperi, Secale cereale, Pygeum africanum, Urtica dioica, Curcubita pepo) met inclusion criteria and were reviewed. Many studies did not report results in a method allowing meta-analysis. Serenoa repens, extracted from the saw palmetto, is the most widely used phytotherapeutic agent for BPH. A total of 18 trials involving 2939 men were reviewed. Compared with men receiving placebo, men taking Serenoa repens reported greater improvement of urinary tract symptoms and flow measures. Serenoa repens decreased nocturia (weighted mean difference $(\mathrm{WMD})=-0.76$ times per evening; $95 \% \mathrm{CI}=-1.22$ to $-0.32 ; n=10$ studies) and improved peak urine flow $\left(\mathrm{WMD}=1.93 \mathrm{ml} \mathrm{s}^{-1} ; 95 \% \mathrm{CI}=0.72\right.$ to $3.14, n=8$ studies $)$. Men treated with Serenoa repens rated greater improvement of their urinary tract symptoms versus men taking placebo (risk ratio of improvement $=1.72 ; 95 \% \mathrm{CI}=1.21$ to $2.44, n=8$ studies). Improvement in symptoms of BPH was comparable to men receiving the finasteride. Hypoxis rooperi ( $n=4$ studies, 519 men) was also demonstrated to be effective in improving symptom scores and flow measures compared with placebo. For the two studies reporting the International Prostate Symptom Score, the WMD was - 4.9 IPSS points ( $95 \% \mathrm{CI}=-6.3$ to $-3.5, n=2$ studies) and the WMD for peak urine flow was $3.91 \mathrm{ml} \mathrm{s}^{-1}$ (95\% CI $=0.91$ to $6.90, n=4$ studies). Secale cereale ( $n=4$ studies, 444 men) was found to modestly improve overall urological symptoms. Pygeum africanum ( $n=17$ studies, 900 men) may be a useful treatment option for BPH. However, review of the literature has found inadequate reporting of outcomes which currently limit the ability to estimate its safety and efficacy. The studies involving Urtica dioica and Curcubita pepo are limited although these agents may be effective combined with other plant extracts such as Serenoa and Pygeum. Adverse events due to phytotherapies were reported to be generally mild and infrequent.

Conclusions: Randomized studies of Serenoa repens, alone or in combination with other plant extracts, have provided the strongest evidence for efficacy and tolerability in treatment of BPH in comparison with other phytotherapies. Serenoa repens appears to be a useful option for improving lower urinary tract symptoms and flow measures. Hypoxis rooperi and Secale cereale also appear to improve $\mathrm{BPH}$ symptoms although the evidence is less strong for these products. Pygeum africanum has been studied extensively but inadequate reporting of outcomes limits the ability to conclusively recommend it. There is no convincing evidence supporting the use of Urtica dioica or Curcubita pepo alone for treatment of BPH. Overall, phytotherapies are less costly, well tolerated and adverse events are generally mild and infrequent. Future randomized controlled trials using standardized preparations of phytotherapeutic agents with longer study durations are needed to determine their long-term effectiveness in the treatment of $\mathrm{BPH}$.
\end{abstract}

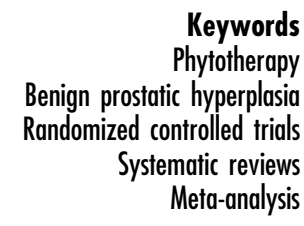

Keywords Phyiotherapy Benign prostatic hyperplasia Systematic reviews Meta-analysis 
Table 1 Plant extracts commonly used for BPH

\begin{tabular}{ll}
\hline Scientific name & Common name \\
\hline Curcubita pepo & Pumpkin seed \\
Hypoxis rooperi & South African star grass \\
Pygeum africanum & African plum tree \\
Serenoa repens [Sabal serrulata] & Saw palmetto berry \\
Urtica dioica & Stinging nettle root \\
Secale cereale & Rye pollen \\
\hline
\end{tabular}

Phytotherapy or the use of plant extracts for treatment of lower urinary tract symptoms (LUTS) consistent with benign prostatic hyperplasia (BPH) was first described in Egypt in the 15 th century $\mathrm{BC}^{1}$. Phytotherapy is common in Europe and is increasing in the Western Hemisphere. In 1998, the sale of botanical medications in the United States was $\$ 1.5$ billion per year and the use of phytotherapeutic compounds increased nearly $70 \%$ among US adults, 2 .

About 30 phytotherapeutic compounds are used for the treatment of $\mathrm{BPH}$ (Table 1). Phytotherapeutic agents represent nearly half the medications dispensed for treatment of BPH in Italy, compared with 5\% for alphablockers and $5 \%$ for $5 \alpha$-reductase inhibitors ${ }^{4}$. In Germany and Austria, phytotherapy is the first-line treatment for mild to moderate lower urinary tract symptoms and represents more than $90 \%$ of drugs prescribed for treatment of BPH. In the United States, phytotherapies for BPH are available as nonprescription dietary supplements. Nearly a quarter of men attending a United States urology clinic who had previously treated BPH indicated they had used phytotherapeutic agents for self-treatment of urinary tract symptoms $s^{5}$

Phytotherapies are often promoted to 'maintain a healthy prostate' and as natural and harmless treatment of BPH symptoms. Despite their popularity with the public there has been reluctance among many practitioners to routinely recommend these products. This is because of uncertainty regarding their efficacy and safety ${ }^{6,7}$. Most phytotherapeutic compounds are unlicensed and do not require evidence of efficacy, safety or purity.

There have been over 40 published randomized controlled trials evaluating the efficacy of phytotherapy for symptomatic BPH in approximately 5000 men. Many more trials are in progress and should provide needed evidence regarding the role of phytotherapeutic products.

Systematic reviews of the existing literature provide a systematic assembly of the results of primary investigations using strategies that limit bias and random error ${ }^{8}$. Systematic reviews efficiently integrate unmanageable amounts of information and provide results that allow for rational decision making. They can establish whether findings are consistent and generalized or whether findings vary by subsets. If clinically and statistically appropriate, a quantitative summary (meta-analysis) can be performed resulting in statistical pooling of results and enhancement of the estimates of therapeutic effects and risk estimates.
This is especially helpful when a large number of small trials have been conducted or when results from comparable studies provide differing results. Systematic reviews also identify gaps in existing evidence and make recommendations for future research to close these scientific and clinical gaps.

\section{Phytotherapeutic compounds}

\section{Serenoa repens (saw palmetto)}

\section{Background}

The most widely used phytotherapeutic agent for $\mathrm{BPH}$ is the extract of the dried ripe fruit from the American dwarf palm plant, saw palmetto, Serenoa repens (also known by its botanical name as Sabal serrulata). Serenoa repens has been approved in France and Germany for treatment of $\mathrm{BPH}$. Berries from saw palmetto were first used by the American Indians in the southeast United States in the early 1700 s to treat testicular atrophy, erectile dysfunction, and prostate gland swelling or inflammation ${ }^{1}$. The medicinal value of Serenoa repens for relief of prostate gland swelling has been reported since the 1800s. The mechanism of action of Serenoa repens has been investigated in several in vitro or indirect in vivo studies and has not been definitively defined. The mechanism may include alteration of cholesterol metabolism, anti-oestrogenic, anti-androgenic (including $5 \alpha$-reductase inhibitor activity), antiinflammatory effects, and a decrease in available sex hormone binding globulin ${ }^{9-12}$.

\section{Results of studies}

A systematic review and meta-analysis of randomized trials assessed the existing evidence regarding efficacy and safety of Serenoa repens in men with symptomatic $\mathrm{BPH}^{13}$. Studies were identified through a search of MEDLINE (1966-1997), EMBASE, Phytodok, the Cochrane Library, bibliographies of identified trials and review articles, and contact with relevant authors and drug companies. Randomized trials were included if participants had symptomatic BPH, the intervention was a preparation of Serenoa repens alone or in combination with other phytotherapeutic agents, a control group received placebo or other pharmacologic therapies for $\mathrm{BPH}$, and the treatment duration was at least 30 days. Two investigators independently extracted key data on design features, subject characteristics, therapy allocation and outcomes of the studies.

A total of 18 studies involving almost 3000 men were identified and analysed ${ }^{14-31}$ (Tables 2-5). Many studies did not report results in a method that permitted quantitative meta-analysis. Sixteen trials were double blinded, 14 were placebo controlled and four involved Serenoa repens in combination with other phytotherapeutic agents. The average study duration was 9 weeks (range 4-48 weeks) and the average age of enrollees was 65 years. Baseline characteristics regarding prostate volume, urine flow rates 


\begin{tabular}{|c|c|c|c|c|c|}
\hline $\begin{array}{l}\text { Comparison: } \\
\text { Outcome: } \\
\text { Study }\end{array}$ & $\begin{array}{c}\text { Serenoa repens versus } \\
\text { Patient Self-rating for In } \\
\text { Serenoa repens } \\
n / N\end{array}$ & $\begin{array}{l}\text { acebo } \\
\text { roved Syn } \\
\text { Placebo } \\
\text { n/N }\end{array}$ & $\begin{array}{c}\text { (\# men rating very } \\
\text { RR } \\
\text { (95\% } \\
\text { (9l Random) }\end{array}$ & $\begin{array}{c}\text { to good) } \\
\text { Weight } \\
y / 4\end{array}$ & $\begin{array}{l}\text { RR } \\
\text { (95\% } \% \text { Random) }\end{array}$ \\
\hline Eraeckman & $84 / 125$ & $62 / 113$ & 世- & 23.6 & $1.22[1.00,1.51]$ \\
\hline Carbin & $22 / 27$ & $3 / 28$ & $\ldots$ & 8.1 & $7.60[2.57,22.49]$ \\
\hline Charnpautt & $44 \int 55$ & 30155 & $\rightarrow$ & 22.4 & $1.47[1,11,1.93]$ \\
\hline Descotes & $58 / 82$ & $63 / 94$ & 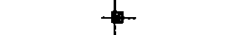 & 23.7 & $1.06[0.87,1.29]$ \\
\hline Mandressi & $18 / 20$ & $8 / 20$ & $\longrightarrow$ & 16.3 & $2.25[1.29,3.92]$ \\
\hline Metzker & $16 / 20$ & $2 / 20$ & & 6.0 & $8.00[2.11,30.34]$ \\
\hline Total(95\%Cl) & $242 / 329$ & $168 / 330$ & $\Rightarrow$ & 100.0 & $1.76[1.21,2.56]$ \\
\hline Chi-square 30.12 & $=5) P: 0.00 \quad Z=2.98 \mathrm{P}:=0.00001$ & & & & \\
\hline
\end{tabular}

Fig. 1 Effect of Serenoa repens on self-rating of improvement in urinary tract symptoms for men treated with Serenoa repens vs. placebo

and symptom scale scores were comparable with previous trials evaluating pharmacologic management of $\mathrm{BPH}$.

The available data indicate that Serenoa repens (alone or in combination with other phytotherapeutic agents) improves urinary symptoms and flow measures (Figs 13). Compared with placebo, saw palmetto improved urinary symptom scores by $28 \%$ and nocturia by $25 \%$ (the weighted mean difference (WMD) $=-0.76$ times per evening; $95 \% \mathrm{CI}=-1.22$ to $-0.32 ; n=10$ studies). Peak urine flow was improved by $24 \%$ (WMD $=1.93 \mathrm{ml} \mathrm{s}^{-1} ; 95 \%$ $\mathrm{CI}=0.72$ to $3.14, n=8$ studies), mean urine flow by $28 \%$ (2.22 $\mathrm{ml} \mathrm{s}^{-1}$; data not shown), and residual urine volume by $43 \%$ ( $-22.05 \mathrm{ml}$; data not shown). Men taking Serenoa repens were more likely to report improvement in urinary symptoms than men taking placebo ( $73.6 \%$ vs. $50.9 \%$; risk ratio $=1.76$ ). Adverse effects were generally mild and comparable with placebo.

Compared with finasteride ${ }^{17,30}$, saw palmetto provided similar responses in urologic symptom scores $(0.37$ International Prostate Symptom Score (IPSS) points), nocturia $(-0.20$ times per evening) and flow measures.
Saw palmetto was associated with a lower rate of erectile dysfunction than finasteride (1.1\% vs. $4.9 \% ; P<0.001)$ and reduced neither prostate size nor prostate specific antigen (PSA) levels. Critics have stated that comparing saw palmetto with finasteride might be showing equivalency to placebo. However, previous trials and meta-analyses have demonstrated that finasteride provides symptomatic improvement in men with prostate glands $>40 \mathrm{~g}$, a size comparable to those enrolled in this study ${ }^{32,33}$.

The treatment effect sizes noted with saw palmetto were comparable to effects reported with other pharmacologic agents, such as finasteride. However, the results should be viewed cautiously. Studies utilized different doses and preparations of Serenoa repens (including combination preparations). The most extensively investigated preparation of Serenoa repens is manufactured in France and sold as Permixon. The most commonly reported dosage was $160 \mathrm{mg}$ twice per day. Many studies did not report outcome data in a consistent fashion. Only three studies reported validated urologic symptom scales. Trials were of short duration with only two studies having follow-up of at least

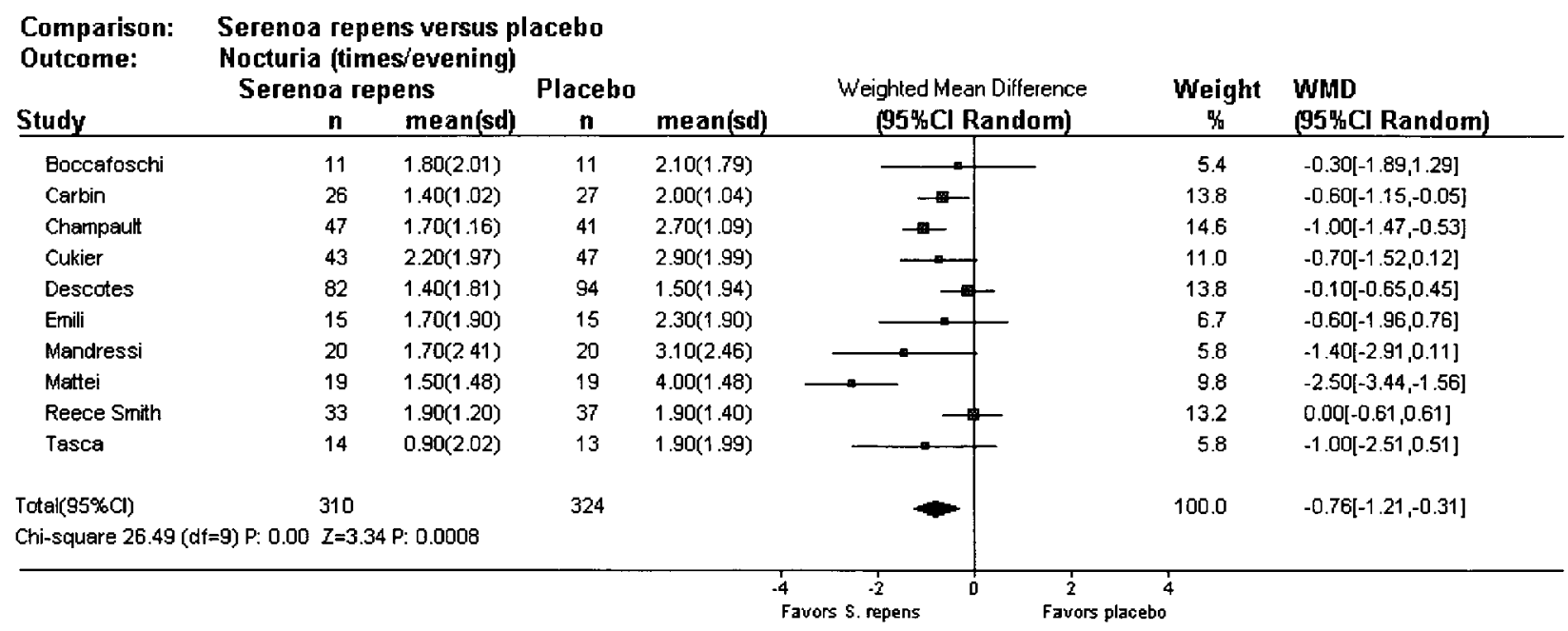

Fig. 2 Effect of Serenoa repens on nocturia for men treated with Serenoa repens vs. placebo 


\begin{tabular}{|c|c|c|c|c|c|c|}
\hline First author & Year & Design & $\begin{array}{l}\text { Sample } \\
\text { size }^{\star}\end{array}$ & $\begin{array}{l}\text { Dose } \\
\text { per day }\end{array}$ & Main outcome measures & Results \\
\hline Boccafoschi & 1983 & $\begin{array}{l}\text { Randomized } \\
\text { controlled trial (RCT); } \\
\text { double-blind (DB); } \\
\text { placebo-controlled (PC). } \\
\text { Study duration (SD): } \\
8.5 \text { wks }\end{array}$ & $22(0)$ & $320 \mathrm{mg}$ & $\begin{array}{l}\text { Nocturia (times/evening); } \\
\text { peak urine flow rate }(\mathrm{ml} / \mathrm{s}) ; \\
\text { mean urine flow rate }(\mathrm{ml} / \mathrm{s}) ; \\
\text { residual volume }(\mathrm{ml})\end{array}$ & $\begin{array}{l}\text { Significant improvements vs. } \\
\text { placebo in nocturia, peak } \\
\text { and mean urine flow } \\
\text { rates }(P<0.05)\end{array}$ \\
\hline Braeckman & 1997 & $\begin{array}{l}\text { RCT; DB; PC. } \\
\text { SD: } 12 \text { wks }\end{array}$ & $238(12)$ & $320 \mathrm{mg}$ & $\begin{array}{l}\text { Symptom scale score (not identified); } \\
\text { patient self-rating of improvement; } \\
\text { peak urine flow; mean urine flow; } \\
\text { residual volume }\end{array}$ & $\begin{array}{l}\text { Significant improvement vs. } \\
\text { placebo in symptoms } \\
\text { improvement } \\
\text { (symptom score) }\end{array}$ \\
\hline Carbin & 1990 & $\begin{array}{l}\text { Sabal combined with } \\
\text { C. pepo L.). RCT; DB; } \\
\text { PC. SD: } 12 \text { wks }\end{array}$ & $55(2)$ & $320 \mathrm{mg}$ & $\begin{array}{l}\text { Patient self-rating of improvement; } \\
\text { nocturia; residual volume }\end{array}$ & $\begin{array}{l}\text { Significant improvements vs. } \\
\text { placebo in self-rating of } \\
\text { improvement }(P<0.001) \\
\text { nocturia }(P<0.01) \\
\text { residual volume }(P<0.001)\end{array}$ \\
\hline Carraro & 1996 & $\begin{array}{l}\text { RCT; DB; } \\
\text { Active-controlled (AC) } \\
\text { (Finasteride } 5 \mathrm{mg} \text { ) } \\
\text { SD: } 26 \text { wks }\end{array}$ & $1098(147)$ & $320 \mathrm{mg}$ & $\begin{array}{l}\text { International Prostate Symptom } \\
\text { Score (IPSS); peak urine flow; } \\
\text { mean urine flow; } \\
\text { prostate volume }\end{array}$ & $\begin{array}{l}\text { Similar improvements in IPSS } \\
\text { and peak urine flow. } \\
\text { Significant decrease in } \\
\text { prostate volume }(P<0.001) \\
\text { with finasteride. Fewer adverse } \\
\text { events associated with } \\
\text { phytotherapy }\end{array}$ \\
\hline Champault & 1984 & $\begin{array}{l}\text { RCT; DB; PC. } \\
\text { SD: } 4 \text { wks }\end{array}$ & $110(16)$ & $160 \mathrm{mg}$ & $\begin{array}{l}\text { Patient self-rating and physician- } \\
\text { rating of improvement; nocturia; } \\
\text { mean urine low }\end{array}$ & $\begin{array}{l}\text { Significant improvements vs. } \\
\text { placebo in all outcomes } \\
(P<0.001)\end{array}$ \\
\hline Cukier & 1985 & $\begin{array}{l}\text { RCT; DB; PC. } \\
\text { SD: } 10 \text { wks }\end{array}$ & $168(22)$ & $320 \mathrm{mg}$ & Nocturia; residual volume & $\begin{array}{l}\text { Significant improvements vs. } \\
\text { placebo in nocturia } \\
(P<0.001) \text {, residual volume } \\
(P<0.05)\end{array}$ \\
\hline Descotes & 1995 & $\begin{array}{l}\text { RCT; DB; PC. } \\
\text { SD: } 4 \text { wks }\end{array}$ & $215(39)$ & $320 \mathrm{mg}$ & $\begin{array}{l}\text { Patient self-rating and physician- } \\
\text { rating of improvement; nocturia; } \\
\text { peak urine flow }\end{array}$ & $\begin{array}{l}\text { Significant improvements vs. } \\
\text { placebo in nocturia, peak } \\
\text { urine flow }(P<0.05)\end{array}$ \\
\hline Emili & 1983 & $\begin{array}{l}\text { RCT; DB; PC. } \\
\text { SD: } 4 \text { wks }\end{array}$ & $30(0)$ & $320 \mathrm{mg}$ & $\begin{array}{l}\text { Nocturia; peak urine flow; residual } \\
\text { volume }\end{array}$ & $\begin{array}{l}\text { Significant improvements vs. } \\
\text { placebo in all outcomes } \\
(P<0.05,0.001 \text { for peak } \\
\text { urine flow })\end{array}$ \\
\hline Gabric & 1987 & $\begin{array}{l}\text { Sabal combined with } \\
\text { Urtica extracts. RCT; } \\
\text { DB; PC. SD: } 6 \text { wks }\end{array}$ & $30(0)$ & $\begin{array}{l}20 \text { drops } \times 3 \\
\text { daily }\end{array}$ & $\begin{array}{l}\text { Physician-rating of improvement; } \\
\text { peak urine flow; } \\
\text { residual volume }\end{array}$ & $\begin{array}{l}\text { Significant improvements vs. } \\
\text { placebo in all outcomes } \\
(P<0.05)\end{array}$ \\
\hline Löbelenz & 1992 & $\begin{array}{l}\text { RCT; DB; PC. } \\
\text { SD: } 6 \text { wks }\end{array}$ & $60(0)$ & $100 \mathrm{mg}$ & Peak urine flow & $\begin{array}{l}\text { No significant improvement } \\
\text { vs. placebo }\end{array}$ \\
\hline Mandressi & 1983 & $\begin{array}{l}\text { 3-arm study (vs. } \\
\text { placebo, vs. Pygeum } \\
\text { extracts). RCT; DB } \\
\text { SD: } 4 \text { wks }\end{array}$ & $60(0)$ & $320 \mathrm{mg}$ & $\begin{array}{l}\text { Patient self-rating of improvement; } \\
\text { nocturia; residual volume }\end{array}$ & $\begin{array}{l}\text { Improvements: patient self- } \\
\text { rating of improvement } \\
\text { Serenoa } 90 \%, \text { Pygeum } \\
60 \% \text {, placebo } 40 \%,(P<0.05 \text {, } \\
P<0.001) \text {; nocturia Serenoa } \\
42 \% \text {, Pygeum } 38 \% \text {, placebo } \\
-4 \% \text {; residual volume } \\
\text { Serenoa } 10 \% \text {, Pygeum }-4 \% \text {, } \\
\text { placebo } 0 \%\end{array}$ \\
\hline
\end{tabular}


Table 2. (continued)

\begin{tabular}{|c|c|c|c|c|c|c|}
\hline First author & Year & Design & $\begin{array}{c}\text { Sample } \\
\text { size }^{\star}\end{array}$ & $\begin{array}{l}\text { Dose } \\
\text { per day }\end{array}$ & Main outcome measures & Results \\
\hline Mattei & 1990 & $\begin{array}{l}\text { RCT; DB; PC } \\
\text { SD: } 13 \text { wks }\end{array}$ & $40(2)$ & $320 \mathrm{mg}$ & Nocturia; residual volume & $\begin{array}{l}\text { Significant improvements vs. } \\
\text { placebo in nocturia }(P<0.05) \\
\text { residual volume }(P<0.01)\end{array}$ \\
\hline Metzker & 1996 & $\begin{array}{l}\text { Sabal combined with } \\
\text { Urtica extracts ( } 120 \\
\text { mg). RCT; DB; PC. } \\
\text { SD: } 48 \text { wks }\end{array}$ & $40(3)$ & $320 \mathrm{mg}$ & $\begin{array}{l}\text { International Prostate Symptom } \\
\text { Score (IPSS); peak urine flow; } \\
\text { residual volume }\end{array}$ & $\begin{array}{l}\text { Significant improvements vs. } \\
\text { placebo in IPSS }(P<0.01) \\
\text { peak urine flow }(P<0.05)\end{array}$ \\
\hline Pannunzio & 1986 & $\begin{array}{l}\mathrm{RCT} ; \mathrm{AC} \\
\text { (Gestonorone } \\
\text { caproate } 200 \mathrm{mg} \text { ) } \\
\text { SD: } 8 \text { wks }\end{array}$ & $60(0)$ & $320 \mathrm{mg}$ & Nocturia; peak urine flow & $\begin{array}{l}\text { Significant improvement vs. } \\
\text { control in peak urine flow } \\
(P<0.05)\end{array}$ \\
\hline Reece Smith & 1986 & $\begin{array}{l}\text { RCT; DC. } \\
\text { SD: } 12 \text { wks }\end{array}$ & $80(10)$ & 320 mg & $\begin{array}{l}\text { Patient self-rating and } \\
\text { physician-rating of improvement; } \\
\text { nocturia; peak urine flow; } \\
\text { residual volume }\end{array}$ & $\begin{array}{l}\text { No significant differences in } \\
\text { outcomes improvement versus } \\
\text { placebo }\end{array}$ \\
\hline Roveda & 1994 & $\begin{array}{l}\text { RCT; Comparison } \\
\text { study (oral vs. rectal } \\
\text { capsules) SD: } 4 \text { wks }\end{array}$ & $30(0)$ & $\begin{array}{l}640 \mathrm{mg} \\
\text { for both }\end{array}$ & $\begin{array}{l}\text { Overall effect of treatment; } \\
\text { residual volume }\end{array}$ & $\begin{array}{l}\text { No significant differences between } \\
\text { two groups demonstrating } \\
\text { bioequivalence }\end{array}$ \\
\hline Sokeland & 1997 & $\begin{array}{l}\text { Sabal combined with } \\
\text { Urtica extracts }(120 \mathrm{mg}) \text {. } \\
\text { RCT; DB; AC } \\
\text { (Finasteride } 5 \mathrm{mg} \text { ) } \\
\text { Study duration: } 12 \mathrm{wks}\end{array}$ & $543(54)$ & $320 \mathrm{mg}$ & $\begin{array}{l}\text { International Prostate Symptom } \\
\text { Score (IPSS); peak urine flow; } \\
\text { residual volume; prostate size }\end{array}$ & $\begin{array}{l}\text { No significant differences in } \\
\text { outcomes improvement vs. } \\
\text { finasteride. Fewer adverse } \\
\text { events associated with } \\
\text { phytotherapy }\end{array}$ \\
\hline Tasca & 1985 & $\begin{array}{l}\text { RCT; DB; PC } \\
\text { Study duration: } 8 \text { wks }\end{array}$ & $30(3)$ & $320 \mathrm{mg}$ & Nocturia; peak urine flow & $\begin{array}{l}\text { Significant improvement vs. placebo in } \\
\text { peak urine flow }(P<0.05)\end{array}$ \\
\hline
\end{tabular}


Table 3 Clinical trials of South African star grass extracts (Hypoxis rooperi/ $\beta$-sitosterols): trials of efficacy

\begin{tabular}{|c|c|c|c|c|c|c|}
\hline First author & Year & Design & $\begin{array}{l}\text { Sample } \\
\text { size }^{*}\end{array}$ & $\begin{array}{l}\text { Dose } \\
\text { per day }\end{array}$ & Main outcome measures & Results \\
\hline Berges & 1995 & $\begin{array}{l}\text { RCT; DB; PC. } \\
\text { SD: } 26 \text { wks }\end{array}$ & $200(10)$ & $60 \mathrm{mg}$ & $\begin{array}{l}\text { International Prostate Symptom Score } \\
\text { (IPSS); Boyarsky Symptom Score; peak } \\
\text { urine flow; residual volume; prostate volume }\end{array}$ & $\begin{array}{l}\text { Significant improvements vs. } \\
\text { placebo in all outcomes } \\
(P<0.01) \text { except prostate volume }\end{array}$ \\
\hline Fischer & 1993 & $\begin{array}{l}\text { RCT; DB; PC. } \\
\text { SD: } 4 \text { wks }\end{array}$ & $80(0)$ & $195 \mathrm{mg}$ & $\begin{array}{l}\text { Patient self-rating and physician-rating } \\
\text { of improvement; nocturia; peak urine flow; } \\
\text { residual volume }\end{array}$ & $\begin{array}{l}\text { Significant improvements vs. } \\
\text { placebo in all outcomes } \\
(P<0.001)\end{array}$ \\
\hline Kadow & 1986 & $\begin{array}{l}\text { RCT; DB; PC. } \\
\text { SD: } 24 \text { wks }\end{array}$ & $62(9)$ & $0.30 \mathrm{mg}^{\dagger}$ & $\begin{array}{l}\text { Peak urine flow; residual volume; prostate } \\
\text { volume }\end{array}$ & $\begin{array}{l}\text { No significant differences in } \\
\text { outcomes improvement vs. } \\
\text { placebo }\end{array}$ \\
\hline Klippel & 1997 & $\begin{array}{l}\text { RCT; DB; PC. } \\
\text { SD: } 26 \text { wks }\end{array}$ & $177(22)$ & $195 \mathrm{mg}$ & $\begin{array}{l}\text { International Prostate Symptom Score } \\
\text { (IPSS); peak urine flow; residual volume }\end{array}$ & $\begin{array}{l}\text { Significant improvements vs. } \\
\text { placebo in all outcomes } \\
(P<0.01)\end{array}$ \\
\hline
\end{tabular}

* (), not available to follow-up.

† Study used pure $\beta$-sitosterol- $\beta$-D-glucoside. In the other three trials the quantity of this derivative was $<5 \%$ of the daily dose of $\beta$-sitosterol.

6 months' duration. Therefore, it is not known whether Serenoa repens prevents long-term complications of $\mathrm{BPH}$ such as acute urinary retention or the need for surgical intervention. The only trial comparing Serenoa repens with alpha-blockers lasted less than 3 weeks, making a comparison impossible. Finally, it is possible that study results were not reported if there were no improvements in symptoms or flow measures (publication bias). There are two placebo-controlled studies involving 298 men that were scheduled for completion in 1998. However, their results have not yet been reported.

\section{Summary}

Extracts from the saw palmetto plant, Serenoa repens, provide modest improvement in urinary symptoms and flow measures. Compared with finasteride Serenoa repens produces similar improvements in symptoms and flow measures, has fewer adverse treatment effects and costs less. The long-term safety and efficacy of Serenoa repens and its ability to prevent complications from $\mathrm{BPH}$ are not known. Standardized preparations are often not available. Publication of ongoing trials is encouraged and initiation of long term studies compared with alpha-blockers would be useful.

\section{Hypoxis rooperi (Soutb African star grass, $\beta$ - sitosterol)}

\section{Background}

Phytosterol extracts derived from the South African star grass, Hypoxis rooperi, are popular. The presumed active constituent is $\beta$-sitosterol. Beta-sitosterol contains a mixture of phytosterols, with smaller amounts of other sterols, bonded with glucosides ${ }^{1}$. Additionally, the quantity of $\beta$ sitosterol- $\beta \mathrm{D}$-glucoside is often reported. The product is sold under the trade names Harzol or Azuprostat. Although the mechanism of action of $\beta$-sitosterols is not known it may be related to cholesterol metabolism or anti-inflammatory effects (via interference with prostaglandin metabolism) ${ }^{1}$.

\section{Results of studies}

Four randomized controlled trials evaluated $\beta$-sitosterol in 519 men with symptomatic $\mathrm{BPH}^{34-37}$ (Table 3). All were

\begin{tabular}{|c|c|c|c|c|c|c|c|}
\hline \multirow{3}{*}{$\begin{array}{l}\text { Comparison: } \\
\text { Outcome: } \\
\text { Study } \\
\text { Boccatoschi }\end{array}$} & \multicolumn{7}{|c|}{$\begin{array}{l}\text { Serenoa repens versus placebo } \\
\text { Peak Urine Flow (mlisec) }\end{array}$} \\
\hline & $\mathbf{n}$ & $\operatorname{mean}(\mathrm{sd})$ & $\begin{array}{c}\text { Placebo } \\
\mathbf{n}\end{array}$ & $\operatorname{mean}(s d)$ & $\begin{array}{l}\text { Weighted Mean Difference } \\
\text { (95\% CI Random) }\end{array}$ & $\begin{array}{c}\text { Weight } \\
\%\end{array}$ & $\begin{array}{l}\text { WhD } \\
\text { (95\% } \% \text { Cl Random) }\end{array}$ \\
\hline & 11 & $13.70(7.03)$ & 11 & $12.20(7.03)$ & $\longrightarrow$ & 4.1 & $1.50[-4.38,7.38]$ \\
\hline Eraeckman & 106 & $13.20(5.76)$ & 99 & $12.20(5.60)$ & - & 45.1 & $1.00[-0.56,2.56]$ \\
\hline Charnpault & 46 & $16.10(19.19)$ & 39 & $10.60(17.67)$ & & $\rightarrow \quad 2.3$ & $5.50[-2.34,13.34]$ \\
\hline Descotes & 82 & $15.30(13.04)$ & 94 & $13.50(13.96)$ & & 8.7 & $1.80[-2.19,5.79]$ \\
\hline Emili & 15 & $13.70(4.37)$ & 15 & $9.40(4.37)$ & - & 13.8 & $4.30[1.17,7.43]$ \\
\hline Gabric & 15 & $14.60(5.57)$ & 14 & $10.80(5.39)$ & & 8.7 & $3.80[-0.19,7.79]$ \\
\hline Reece Smith & 33 & $8.50(7.12)$ & 37 & $8.60(7.12)$ & & 12.2 & $-0.10[-3.44,3.24]$ \\
\hline Tasca & 14 & $16.20(7.03)$ & 13 & $11.80(7.03)$ & + & 5.0 & $4.40[-0.91,9.71]$ \\
\hline Total( $95 \% \mathrm{Cl})$ & 322 & & 322 & & & 100.0 & $1.93[0.72,3.14]$ \\
\hline Chi-square 7.46 ( & 7) $P: 0.38 \quad Z=3.1$ & P: 0.002 & & & & & \\
\hline
\end{tabular}

Fig. 3 Effect of Serenoa repens on peak urine flow for men treated with Serenoa repens vs. placebo 
Table 4 Clinical trials of rye grass pollen extracts (Secale cereale): trials of efficacy

\begin{tabular}{|c|c|c|c|c|c|c|}
\hline First author & Year & Design & Sample size* & Dose & Main outcome measures & Results \\
\hline Becker & 1988 & $\begin{array}{l}\text { RCT; DB; PC. } \\
\text { SD: } 12 \text { wks }\end{array}$ & $103(7)$ & $\mathrm{NA}^{\dagger}$ & $\begin{array}{l}\text { Nocturia; peak urine flow; residual } \\
\text { volume }\end{array}$ & $\begin{array}{l}\text { Significant improvements vs. } \\
\text { placebo in nocturia }(P<0.01) \text {, } \\
\text { residual volume }(P<0.05)\end{array}$ \\
\hline Buck & 1990 & $\begin{array}{l}\text { RCT; DB; PC. } \\
\text { SD: } 24 \text { wks }\end{array}$ & $60(7)$ & NA & $\begin{array}{l}\text { 'Overall improvement in symptoms'; } \\
\text { nocturia; peak urine flow; residual } \\
\text { volume }\end{array}$ & $\begin{array}{l}\text { Significant improvements vs. } \\
\text { placebo in overall improvement in } \\
\text { symptoms }(P<0.01) \text {, residual } \\
\text { volume }(P<0.05)\end{array}$ \\
\hline Dutkiewicz & 1996 & $\begin{array}{l}\text { Controlled clinical trial } \\
\text { (CCT); AC (Pygeum } \\
\text { extracts 'Tadenan') } \\
\text { SD: } 16 \text { wks }\end{array}$ & $89(0)$ & NA & $\begin{array}{l}\text { Obstructive and Irritative symptom } \\
\text { scores; peak urine flow; residual } \\
\text { volume }\end{array}$ & $\begin{array}{l}\text { Improvements: obstructive symptom } \\
\text { score Secale } 63 \% \text {, Pygeum } 46 \% \text {; } \\
\text { irritative symptom score Secale } \\
68 \% \text {, Pygeum } 40 \% \text {; peak urine flow } \\
\text { Secale } 20 \% \text {, Pygeum } 11 \% \text {; residual } \\
\text { volume Secale } 48 \% \text {, Pygeum } 22 \%\end{array}$ \\
\hline Maekawa & 1981 & $\begin{array}{l}\text { RCT; DB; AC. } \\
\text { (Paraprost } 6 \mathrm{~g}) \\
\text { SD } 12 \text { wks }\end{array}$ & $192(14)$ & $252 \mathrm{mg}$ & $\begin{array}{l}\text { International Prostate Symptom } \\
\text { Score (IPSS); nocturia; peak } \\
\text { urine flow; residual volume; } \\
\text { prostate size }\end{array}$ & $\begin{array}{l}\text { No significant differences in } \\
\text { outcomes improvement vs. control }\end{array}$ \\
\hline
\end{tabular}

* (), not available to follow-up.

† NA, not available.

double-blinded and lasted between 4 and 26 weeks. Three trials used non-glucosidic $\beta$-sitosterols in doses ranging from $30 \mathrm{mg}$ to greater than $120 \mathrm{mg}$ per day ${ }^{34,35,37}$. The other trial utilized a preparation that contained $100 \% \beta$ sitosteryl- $\beta$-D-glucoside $(0.15 \mathrm{mg} \text { twice a day })^{36}$. The average age of participants was 65 years. Men had moderately severe BPH (mean baseline IPSS score $=15.2$; peak urine flow $=10.2 \mathrm{ml} \mathrm{s}^{-1}$; prostate size $=49 \mathrm{cc}$ ).

Beta-sitosterol provided statistically significant improvements in urinary symptom scores and flow measures (Figs 4 and 5). In the two studies reporting the IPSS score, the WMD compared with placebo was -4.9 points $(95 \% \mathrm{CI}=$ -6.3 to $-3.5, n=2$ studies) (35\% improvement). The WMD for peak urine flow was $3.91 \mathrm{ml} \mathrm{s}^{-1}$ (45\% improvement) and for residual volume the WMD $=-28.62 \mathrm{ml}(95 \%$ CI $=0.91-6.90, n=4$ studies) (29\% improvement). Betasitosterol did not reduce prostate size and the trial using $100 \% \beta$-sitosteryl- $\beta$-D-glucoside (WA184) did not show improvement in urinary flow rates. Adverse events were infrequent and mild. Withdrawal rates were less than 10\% and did not differ from placebo.

\section{Summary}

An extract from South African star grass, $\beta$-sitosterol, improved urologic symptoms and flow measures. However, the existing evidence is limited to trials of short duration, relatively few patients studied and lack of standardized $\beta$-sitosterol preparations. Their long term effectiveness, safety and ability to prevent BPH complications are not known.

\section{Secale cereale (rye-grass pollen)}

\section{Background}

Rye pollen extract is prepared from the rye-grass, Secale cereale. It is used by millions of men worldwide and is a registered pharmaceutical throughout Western Europe, Japan, Korea and Argentina ${ }^{38}$. In the United States, Cernilton is used as a nutritional supplement by approximately 5000 men $^{39}$. One dose contains $60 \mathrm{mg}$ of Cernitin T60, a water-soluble pollen extract fraction, and $3 \mathrm{mg}$ of Cernitin GBX, an acetone-soluble pollen extract fraction ${ }^{38}$. The acetone-soluble fraction was found to contain $\beta$ sterols ${ }^{40}$. In vitro studies suggest that Cernilton may have anti-androgenic effects, relax urethral smooth muscle tone and increase bladder muscle contraction, or may act on the alpha-adrenergic receptors and relax the internal and external sphincter muscles ${ }^{41-43}$.

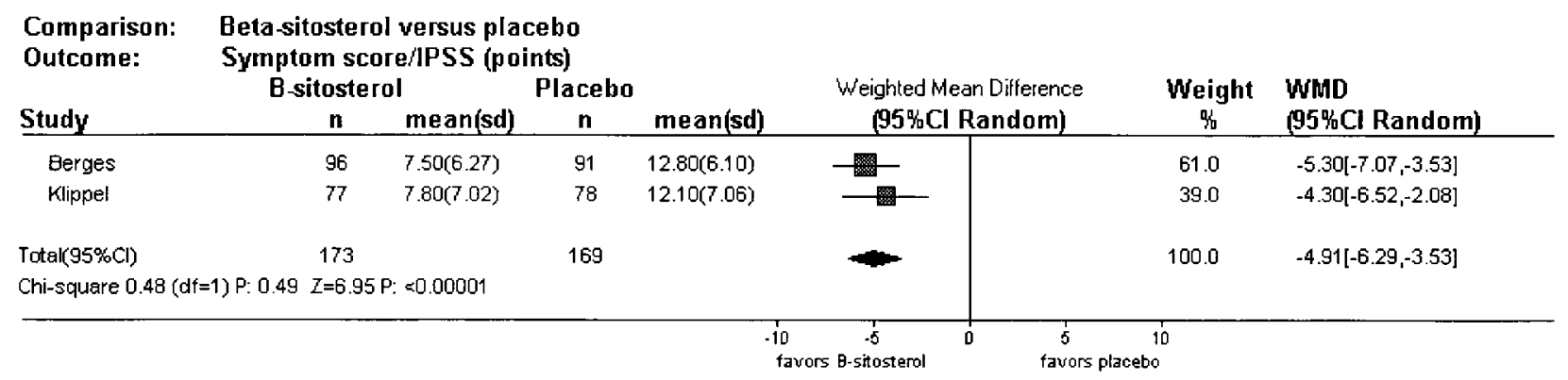

Fig. 4 Effect of $\beta$-sitosterol on urinary symptom scores vs. placebo 
Table 5 Clinical trials of African plum tree extracts (Pygeum africanum): trials of efficacy

\begin{tabular}{|c|c|c|c|c|c|c|}
\hline First author & Year & Design & $\begin{array}{l}\text { Sample } \\
\text { size }^{\star}\end{array}$ & $\begin{array}{l}\text { Dose } \\
\text { per day }\end{array}$ & Main outcome measures & Results \\
\hline Barlet & 1990 & $\begin{array}{l}\text { RCT; DB; PC } \\
\text { SD: } 8 \text { wks }\end{array}$ & $263(8)$ & $100 \mathrm{mg}$ & $\begin{array}{l}\text { Patient self-rating and physician- } \\
\text { rating of improvement; nocturia; } \\
\text { peak urine flow; residual volume }\end{array}$ & $\begin{array}{l}\text { Significant improvements vs. placebo } \\
\text { in all outcomes }(P<0.01)\end{array}$ \\
\hline Barth & 1981 & $\begin{array}{l}3 \text { combined studies: } \\
\text { Pygeum vs. (1) } \\
\text { Placebo, (2) Sitosterin, } \\
\text { and (3) Urtica extracts. } \\
\text { RCT; DB; PC; AC } \\
\text { SD: } 8 \text { wks }\end{array}$ & $\begin{array}{l}96(43) \\
71(18) \\
48(6)\end{array}$ & $100 \mathrm{mg}$ & $\begin{array}{l}\text { Nocturia; peak urine flow; } \\
\text { residual volume }\end{array}$ & $\begin{array}{l}\text { Improvements: Nocturia Pygeum and } \\
\text { placebo 'reduced almost to normal'; } \\
\text { peak urine flow Pygeum } 8 \% \text {, } \\
\text { placebo } 10 \% \text {; residual volume } \\
\text { Pygeum } 48 \% \text {, placebo } 37 \% \text {. } \\
\text { Pygeum reduced nocturia, peak urine } \\
\text { flow, and residual volume>controls }\end{array}$ \\
\hline Bassi & 1987 & $\begin{array}{l}\text { RCT; DB; PC } \\
\text { SD: } 8 \text { wks }\end{array}$ & $40(0)$ & $100 \mathrm{mg}$ & Nocturia; peak urine flow & $\begin{array}{l}\text { Significant improvements vs. } \\
\text { placebo in nocturia }(P<0.001) \\
\text { peak urine flow }(P<0.05)\end{array}$ \\
\hline Blitz & 1985 & $\begin{array}{l}\text { RCT; DB; PC } \\
\text { SD: } 6 \text { wks }\end{array}$ & $57(0)$ & $100 \mathrm{mg}$ & $\begin{array}{l}\text { 'Overall improvement in } \\
\text { symptoms' }\end{array}$ & $\begin{array}{l}\text { Significant improvements vs. placebo } \\
\text { in overall improvement in symptoms } \\
(P<0.05)\end{array}$ \\
\hline Bongi & 1972 & $\begin{array}{l}\text { RCT; DB; PC } \\
\text { SD: } 8 \text { wks }\end{array}$ & $50(0)$ & $75 \mathrm{mg}$ & $\begin{array}{l}\text { 'Overall improvement in } \\
\text { symptoms'; nocturia; } \\
\text { residual volume }\end{array}$ & $\begin{array}{l}\text { Significant improvements vs. } \\
\text { placebo in all outcomes }(P<0.01)\end{array}$ \\
\hline Chatelain & 1999 & $\begin{array}{l}\text { Comparison study of } 2 \\
\text { doses. RCT; DB } \\
\text { SD: } 8 \text { wks }\end{array}$ & $235(26)$ & $\begin{array}{l}50 \mathrm{mg} \\
\times 2 \mathrm{vs} \\
100 \mathrm{mg}\end{array}$ & $\begin{array}{l}\text { International Prostate Symptom } \\
\text { Score (IPSS); peak urine flow }\end{array}$ & $\begin{array}{l}\text { No significant differences between } \\
\text { two groups }\end{array}$ \\
\hline Donkervoort & 1977 & $\begin{array}{l}\text { RCT; DB; PC } \\
\text { SD: } 12 \text { wks }\end{array}$ & $20(4)$ & $100 \mathrm{mg}$ & $\begin{array}{l}\text { 'Overall improvement in } \\
\text { symptoms'; nocturia; peak } \\
\text { urine flow }\end{array}$ & $\begin{array}{l}\text { No significant differences in outcomes } \\
\text { improvement versus placebo }\end{array}$ \\
\hline Dufour & 1984 & $\begin{array}{l}\text { RCT; DB; PC } \\
\text { SD: } 6 \text { wks }\end{array}$ & $120(54)$ & $100 \mathrm{mg}$ & Nocturia & $\begin{array}{l}\text { Significant improvements vs. placebo } \\
\text { in nocturia }(P<0.01)\end{array}$ \\
\hline Dutkiewicz & 1996 & $\begin{array}{l}\text { Controlled clinical trial } \\
\text { (CCT); AC (Secale } \\
\text { extracts 'Cernilton') } \\
\text { SD: } 16 \text { wks }\end{array}$ & $89(0)$ & $\mathrm{NA}^{\dagger}$ & $\begin{array}{l}\text { Obstructive and irritative } \\
\text { symptom scores; peak urine } \\
\text { flow; residual volume }\end{array}$ & $\begin{array}{l}\text { Improvements: obstructive symptom } \\
\text { score Pygeum } 46 \% \text {, Secale } 63 \% \text {; } \\
\text { irritative symptom score Pygeum } 40 \% \text {, } \\
\text { Secale } 68 \% \text {; peak urine flow Pygeum } \\
11 \% \text {, Secale } 20 \% \text {; residual volume } \\
\text { Pygeum } 22 \% \text {, Secale } 48 \%\end{array}$ \\
\hline Frasseto & 1986 & $\begin{array}{l}\text { RCT; DB; PC } \\
\text { SD: } 8 \text { wks }\end{array}$ & $20(0)$ & $200 \mathrm{mg}$ & Nocturia & $\begin{array}{l}\text { Improvement: nocturia Pygeum } 57 \% \text {, } \\
\text { placebo } 19 \%\end{array}$ \\
\hline Gagliardi & & $\begin{array}{l}\text { RCT; DB; AC (control } \\
\text { not identified). } \\
\text { SD: } 4 \text { wks }\end{array}$ & $40(0)$ & $100 \mathrm{mg}$ & Nocturia; residual volume & $\begin{array}{l}\text { Improvements: nocturia Pygeum } 60 \% \text {, } \\
\text { control } 0 \% \text {; residual volume } \\
\text { Pygeum } 71 \% \text {, control } 11 \%\end{array}$ \\
\hline Giacobini & 1986 & $\begin{array}{l}\text { 3-arm study (vs. } \\
\text { Pygeum combined } \\
\text { with medroxy- } \\
\text { progesterone acetate } \\
\text { [Falutal] vs. placebo) } \\
\text { RCT; DB; PC. } \\
\text { SD: } 13 \text { wks }\end{array}$ & $21(0)$ & $200 \mathrm{mg}$ & $\begin{array}{l}\text { Peak urine flow; } \\
\text { residual volume }\end{array}$ & $\begin{array}{l}\text { Improvements: peak urine flow } \\
\text { Pygeum } 28 \% \text {, Pygeum+Farlutal } 39 \% \text {, } \\
\text { placebo } 16 \% \text {; residual volume } \\
\text { Pygeum } 67 \% \text {, Pygeum+Farlutal } 22 \% \text {, } \\
\text { placebo } 0 \%\end{array}$ \\
\hline
\end{tabular}


Table 5. (continued)

\begin{tabular}{|c|c|c|c|c|c|c|}
\hline First author & Year & Design & $\begin{array}{c}\text { Sample } \\
\text { size }^{\star}\end{array}$ & $\begin{array}{c}\text { Dose } \\
\text { per day }\end{array}$ & Main outcome measures & Results \\
\hline Krzeski & 1993 & $\begin{array}{l}\text { Pygeum combined } \\
\text { with Urtica extracts } \\
\text { (600 mg \& } 300 \mathrm{mg} \text { ). } \\
\text { Comparison study of } 2\end{array}$ & $144(19)$ & $\begin{array}{l}50 \mathrm{mg} \\
\text { vs. } 25 \mathrm{mg}\end{array}$ & $\begin{array}{l}\text { 'Overall improvement in } \\
\text { symptoms'; nocturia; } \\
\text { peak urine flow; } \\
\text { residual volume }\end{array}$ & $\begin{array}{l}\text { No significant differences between } \\
\text { two groups }\end{array}$ \\
\hline
\end{tabular}

doses. RCT; DB

SD: 8 wks

Mandressi

1983

3-arm study (vs.

placebo, vs. Serenoa

$60(0)$

residual volume

SD: 4 wks

Patient self-rating of

improvement;

nocturia; residual volume

Improvements: patient self-rating of

improvement Pygeum 60\%,

Serenoa $90 \%$, placebo $40 \%$

(Serenoa vs. Pygeum $P<0.05$

Serenoa vs. placebo $P<0.001$ )

nocturia Pygeum 38\%; Serenoa 42\%,

placebo $-4 \%$; residual volume

Serenoa $10 \%$, Pygeum -4\%,

placebo $0 \%$;

Significant improvements vs. placebo

both outcomes $(P<0.01)$

Significant improvement vs. placebo

in peak urine flow $(P<0.01)$

RCT; DB; PC
SD: 8 wks
RCT; DB; PC
SD: 8 wks
RCT; DB; AC (NSAID).
SD: 8 wks
RCT; DB; PC
SD: 8 wks

Nocturia; peak urine flow

Rigatti

1983

$49(0)$

Residual volume

Rizzo

1985

SD: 8 wks

$40(0) \quad 200 \mathrm{mg}$

Nocturia; peak urine flow;

Significant improvement vs. control in

residual volume $(P<0.05)$

Significant improvement vs. placebo

in nocturia $(P<0.01)$

* (), not available to follow-up.

+ NA, not available. 


\begin{tabular}{|c|c|c|c|c|c|c|c|}
\hline \multirow{2}{*}{$\begin{array}{l}\text { Comparison: } \\
\text { Outcome: } \\
\text { Study }\end{array}$} & \multicolumn{7}{|c|}{$\begin{array}{l}\text { Beta-sitosterol versus placebo } \\
\text { Peak urine flow (m/s/s) }\end{array}$} \\
\hline & $\mathbf{n}$ & mean(sd) & $\begin{array}{c}\text { Placebo } \\
\text { n }\end{array}$ & mean(sd) & $\begin{array}{c}\text { Weighted Mean Difference } \\
\text { (95\%Cl Random) }\end{array}$ & $\begin{array}{c}\text { Weight } \\
\%\end{array}$ & $\begin{array}{l}\text { WMD } \\
\text { (95\% CI Random) }\end{array}$ \\
\hline Berges & 95 & $15.20(6.43)$ & 91 & $11.40(6.30)$ & - & 27.1 & $3.80[1.97,5.63]$ \\
\hline Fischer & 40 & $23.10(7.08)$ & 40 & $14.70(7.08)$ & & $\Rightarrow 22.8$ & $8.40[5.30,11.50]$ \\
\hline Kadow & 25 & $10.75(3.50)$ & 28 & $10.37(3.70)$ & -1 & 26.7 & $0.38[-1.56,2.32]$ \\
\hline Klippel & 77 & $19.40(9.21)$ & 78 & $15.70(9.27)$ & $\longrightarrow$ & 23.4 & $3.70[0.79,6.61]$ \\
\hline Total $(95 \% \mathrm{Cl})$ & 237 & & 237 & & - & 100.0 & $3.91[0.91,6.90]$ \\
\hline \multicolumn{8}{|c|}{ Chi-square $19.43(\mathrm{df}=3) \mathrm{P}: 0.00 \quad Z=2.56 \mathrm{P}: 0.01$} \\
\hline
\end{tabular}

Fig. 5 Effect of $\beta$-sitosterol on peak urine flow vs. placebo

\section{Results of studies}

A total of 444 men have been enrolled in two placebocontrolled $(n=163)$ and two comparative trials lasting from 12 to 24 weeks $^{44-47}$ (Table 4 ). Three studies were double-blinded $^{44,45,47}$. The mean age of participants was 69 years. Differences in the control agents and methods of reporting results did not permit all studies to be combined in a meta-analysis. However, three studies reported symptom scores or measured symptom improvement ${ }^{45-47}$. Nocturia was reported in three studies $^{44,45,47}$ and all studies reported peak urine flow and residual urine volume. Data from all studies were consistent with improvement in symptoms and urinary flow.

Cernilton improved 'self-rated urinary symptoms' versus placebo and Tadenan, an extract from Pygeum africanum $^{46}$. Almost 70\% men taking Cernilton reported symptom improvement compared with 29\% taking placebo. Obstructive and irritative symptom scores improved from baseline by $60 \%$ in men taking Cernilton compared with $40 \%$ with Tadenan.

Cernilton reduced nocturia compared with placebo and Paraprost, a pharmacologic treatment used primarily in Japan containing $265 \mathrm{mg}$ of L-glutamic acid, $100 \mathrm{mg}$ of Lalanine and $45 \mathrm{mg}$ of aminoacetic acid ${ }^{47}$. Versus placebo, there was a two-fold improvement in the percentage of men reporting improvement in nocturia (63\% vs. 31\%) ${ }^{44,45}$. Compared with Paraprost, Cernilton reduced nocturia by 0.40 times per evening. The only adverse event reported was mild nausea.

Although the results suggest that Cernilton provided modest benefit there are limitations to the evidence. The longest treatment duration was 24 weeks. Only one study reported results from a standardized and validated urologic symptom scale. While the manufacturer suggests two to four tablets or capsules daily, the dosages and standardization of preparation were not usually reported. The most frequently reported amount was two Cernilton capsules three times per day.

\section{Summary}

The evidence suggests that an extract from rye-grass pollen,
Cernilton, is well tolerated and modestly improves urologic symptoms. However, trials were of short duration, enrolled relatively few patients, and lacked standard product preparation. Additionally, there was infrequent use of validated symptom scale scores. It does not improve urinary flow measures and the long-term safety and effectiveness is not known.

\section{Pygeum africanum (African plum)}

\section{Background}

Traditionally, the bark of the African plum tree (Pygeum africanum) was collected and powdered, then drunk as a tea to improve genito-urinary symptoms. Purified bark extracts have been used throughout Europe for the past 30 years. The postulated active components include phytosterols, especially $\beta$-sitosterols, pentacyclic triterpenoids and esters of long-chain fatty alcohols. Pygeum africanum extract may suppress LUTS by reducing bladder hyperreactivity, decreasing inflammation, and protecting against abnormal prostate growth ${ }^{48}$.

A 1995 review identified 12 double-blind, placebo controlled studies involving 717 men with $\mathrm{BPH}^{46,49-63}$ (Table 5). Most studies used a Pygeum extract under the trade name Tadenan with doses ranging from 75 to $200 \mathrm{mg} \mathrm{day}^{-1}$. All studies were at least 16 weeks in duration. More than half the studies measured peak urinary flow and all but one measured urinary frequency. Standardized and validated symptom scores were not utilized and there was no pooled estimate of treatment effect size or adverse events. The majority of studies noted an improvement in nocturia compared with placebo. An ongoing double-blind placebo-controlled study is evaluating Tadenan (100 mg and $400 \mathrm{mg}$ ) in 750 men with symptomatic BPH. The primary endpoint is a mean reduction in the IPSS score between baseline and 6 months. However, the results have not been reported.

In five small-scale studies involving 183 men, $P$. africanum was compared with active drug or therapy ${ }^{50,57,63}$. Two studies involved plant extracts (sitosterin and extract of Radix urticae urtae $)^{50}$. The results 
indicate that Pygeum reduced nocturia more than comparators in the 3 studies reporting this endpoint. However, in two of these studies there were no statistical comparisons. Since the publication of this review there have been two additional trials utilizing Pygeum. One was a study utilizing a combination of Pygeum with Urtica and is discussed in the section on Urtica ${ }^{59}$. The other trial demonstrated that Pygeum was less effective than Cernilton in improving 'self-rated urinary symptoms' ${ }^{46}$. Obstructive and irritative symptom scores improved from baseline by $60 \%$ in men taking Cernilton compared with $40 \%$ in men taking Tadenan.

\section{Summary}

Extracts from the African plum tree, Pygeum africanum, may be a useful treatment option for BPH. However, inadequacies in the reporting of outcomes limit the ability to estimate its safety and efficacy. An ongoing trial should provide much needed information on the short-term effectiveness and tolerability of Pygeum africanum.

\section{Urtica dioica (stinging nettle)}

\section{Background}

Extracts from roots of the stinging nettle are often used in Germany for the treatment of BPH. The extracts contain a mixture of water- and alcohol-soluble compounds with extraction procedures varying from company to company. Proposed mechanisms of action include inhibition of prostatic growth factor including blocking the conversion of testosterone to dihydrostersterone ${ }^{1}$.

\section{Results of studies}

There have been five randomized trials evaluating stinging nettle. Three of these involved combinations with other phytotherapeutic agents (Pygeum and Sabal), making it difficult to evaluate the efficacy of stinging nettle alone $^{26,30,59}$. Furthermore, one of these studies merely compared two different doses of a combined extract of Urtica and Pygeum ${ }^{59}$. The report by Sokeland compared a combination of Sabal and Urtica (PRO 160/120) extract with finasteride and placebo ${ }^{30}$. This trial lasted 12 weeks and evaluated 543 men. Compared with finasteride there were no differences in IPSS scores ( -4.8 vs. -5.8 IPSS points), peak urine flow or residual urine volume. More adverse events were associated with finasteride, including more cases of erectile dysfunction, diminished ejaculation volume, and headaches. Compared with placebo, the combination of Sabal-Urtica (Prostagutt) improved IPSS scores by $17 \%(-3.5 \text { IPSS points })^{26}$.

One placebo-controlled study lasting 3 months compared a liquid preparation of stinging nettle with placebo in 41 men with $\mathrm{BPH}^{64}$. An improvement in IPSS scores was noted in men taking stinging nettle. However, because of unacceptable taste this preparation has been removed from the market. Another placebo-controlled trial examined the effectiveness of Urticae extract capsules ${ }^{65}$. Although improvements in peak urine flow and total voided volume were reported, there was no difference in urologic symptoms. Additionally, 24\% of men (6/25) taking Urticae withdrew from the study; half of them due to unspecified side effects.

\section{Summary}

Evidence from randomized trials suggests combination preparations of Urticae appear to provide some benefit for treatment of lower urinary tract symptoms, although stinging nettle extracts alone do not appear to be beneficial. Additional randomized controlled trials need to be conducted before Urticae can be recommended as an effective option for the treatment of LUTS.

\section{Curcubita pepo (pumpkin seed)}

\section{Results of studies}

There has been only one small-scale randomized trial of short duration that has evaluated the efficacy of pumpkin seed extracts ${ }^{16}$. This study evaluated 55 men, lasted for 12 weeks and utilized a preparation that included pumpkin seed, Curcubita pepo, and Sabal serrulata (Curbicin $160 \mathrm{mg}$ three times a day). Compared with placebo, Curbicin improved self-rating of urinary symptoms $(85 \%$ noted improvement vs. $11 \%$ taking placebo) and nocturia. Residual urine volume was reduced by 31\% (42.5 cc) compared with only 6.5\% (7.6 cc) with placebo. Because the study utilized a combination preparation the reported improvement in urologic symptoms and flow measures cannot be clearly attributed to pumpkin seeds.

\section{Summary}

There is no convincing evidence that extracts of pumpkin seed alone improve urologic symptoms or flow measures. They may provide improvement in urinary symptoms and flow measures when used in combination with Sabal serrulata. Randomized controlled trials need to be conducted.

\section{Recommendations and conclusions}

Should physicians recommend plant extracts for treatment of BPH? Despite their popularity and the existence of over 40 randomized controlled trials involving nearly 5000 men, the available data do not yet provide clear evidence of efficacy for most phytotherapeutic products. Extracts of saw palmetto (Serenoa repens) (alone or in combination with other phytotherapeutic products) have the strongest evidence for efficacy and tolerability. They appear to be a useful option for improving lower urinary tract symptoms and flow measures.

Rye-grass pollen (Secale cereale) and South African star grass (Hypoxis rooperi, $\beta$-sitosterol) also appear to improve symptoms and are well tolerated. However, the evidence is 
less strong for these products. African plum tree bark (Pygeum africanum) has been studied extensively but inadequate reporting of outcomes limits the ability to conclusively recommend it. There is no convincing evidence supporting the use of pumpkin seed (Curcubita pepo) or stinging nettle (Urtica dioica) extracts alone for treatment of BPH. They may be effective in combination with other phytotherapeutic products.

The widespread use of phytotherapy attests to the popularity of plant extracts for treatment of BPH symptoms. They cost less and are better tolerated, at least in the shortterm, than either alpha-blockers or finasteride. However, if the primary goal is to reduce symptoms, alpha-blockers such as doxazosin, tamsulosin, alfuzosin or terazosin seem to be a better choice than finasteride and probably phytotherapy. Additionally, plant extracts have not yet been demonstrated to reduce complications from $\mathrm{BPH}$ or the need for surgical intervention in comparison with interventions such as finasteride ${ }^{33}$.

The Committee on Other Medical Therapies of the Fourth International Consultation on BPH concluded that: most plant extract preparations have different components; it is not known what mechanisms of action demonstrated in vitro might be responsible for clinical effects; short-term randomized studies suggest clinical efficacy for some preparations; and studies were usually inadequate due to the methodology utilized, small numbers and short duration of study. Of greatest importance is the completion of additional high quality studies of long duration to fully evaluate the efficacy and safety of phytotherapeutic products for treatment of $\mathrm{BPH}^{6}$.

Until completion of these studies and/or regulation of these products the lack of universal definitions, practices, and standards within the supplement industry place the onus on the physician to judge product quality and efficacy. Manufacturers/companies of plant extracts often use different extraction processes. There is no evidence that the extract from one manufacturer is equivalent to that of another. Additionally, since the active ingredient(s) are not known, it is possible that one product might have clinical efficacy while another does not. Each company's product must be tested to evaluate clinical efficacy and bioactivity.

The following recommendations have been made for assessing quality measures (these do not directly address clinical efficacy or safety) in selecting high-quality and reliable preparations of phytotherapeutic products manufactured in the United States ${ }^{66}$.

1. The manufacturer tests raw ingredients for purity and potency prior to inclusion in a product.

2. The product is manufactured in a pharmaceutically licensed facility registered with the Food and Drug Administration.

3. The product's ingredients meet the applicable United States Pharmacopoeia (USP) standards.
4. All finished products are analysed for purity and potency following production by an independent laboratory using established methods to ensure that the product meets label claims and is of good quality.

In some cases, this information can be found on product labelling. All reputable manufacturers will keep certificates of laboratory results for each finished batch of product on file. These should be available to physicians and pharmacists on request.

\section{References}

1 Lowe FC, Ku JC. Phytotherapy in treatment of benign prostatic hyperplasia: a critical review. Urology 1996; 48: 12-20.

2 Int. Med. World Report, February 1998, Vol. 13, No. 3, 8 pp.

3 Ernst E. Harmless herbs? A review of the recent literature. Am. J. Med. 1998; 104: 170-8.

4 Di Silverio F, Flammia GP, Sciarra A, Caponera M, Mauro M, Buscarini $\mathrm{M}$, et al. Plant extracts in benign prostatic hyperplasia. Minerva Urol. Nefrol. 1993; 45: 143-9.

5 Bales G, Christiano AP, Kirsh E, Gerber GS. Phytotherapeutic agents in the treatment of lower urinary tract symptoms: a demographic analysis of awareness and use at the University of Chicago. Urology 1999; 54: 86-89.

6 Lowe FC, Dreikorn K, Borkowski A, Braeckman J, Denis L, Ferrari $\mathrm{P}$, et al. Review of recent placebo-controlled trials utilizing phytotherapeutic agents for treatment of $\mathrm{BPH}$. Prostate 1998; 37: 187-93.

7 Fitzpatrick JM. Phytotherapy for treatment of benign prostatic hyperplasia: case not proven. Urology 1999; 53: 462-4.

8 Mulrow CD. Rationale for systematic reviews. BMJ 1994; 309 597-9.

9 Dreikorn K, Richter R. Conservative nonhormonal treatment of patients with benign prostatic hyperplasia. In: Ackerman R, Schroeder FH, eds. New Developments in Biosciences 5, Prostatic Hyperplasia. Berlin: Walter de Gruyter \& Co, 1989: 109-31.

10 Marwick C. Growing use of medicinal botanicals forces assessment by drug regulators. JAMA 1995; 273: 607-9.

11 McGuire E. Detrusor response to obstruction. NIH Publication No. 87-2881, Department of Health and Human Services, Rockville, MD, 1987: 221

12 Di Silverio F, D'Eramo G, Lubrano C, Flammia GP, Sciarra A, Palma E, et al. Evidence that Serenoa repens extract displays an antiestrogenic activity in prostatic tissue of benign prostatic hypertrophy patients. Eur. Urol. 1992; 2: 309-14.

13 Wilt TJ, Ishani A, Stark G, MacDonald R, Lau J, Mulrow C. Saw palmetto extracts for treatment of benign prostatic hyperplasia: a systematic review. JAMA 1998; 280: 1604-9.

14 Boccafoschi C, Annoscia S. Confronto fra estratto di Serenoa repens e placebo mediate prova clinica controllata in pazienti con adenomatosi prostatica. Urologia 1983; 50: 1257-68.

15 Braeckman J, Denis L, de Lavel J, Keuppens F, Cornet A, De Bruyne R, et al. A double-blind, placebo-controlled study of the plant extract Serenoa repens in the treatment of benign hyperplasia of the prostate. Eur. J. Clin. Res. 1997; 9: 247-59.

16 Carbin BE, Larsson B, Lindahl O. Treatment of benign prostatic hyperplasia with phytosterols. Br. J. Urol. 1990; 66: 639-41.

17 Carraro JC, Raynaud JP, Koch G, Chisholm GD, Di Silverio F, Teillac P, et al. Comparison of phytotherapy (Permixon) with finasteride in the treatment of benign prostate hyperplasia: a randomized international study of 1098 patients. Prostate 1996; 29: $231-40$.

18 Champault G, Patel JC, Bonnard AM. A double-blind trial of an extract of the plant Serenoa repens in benign prostatic hyperplasia. Br. J. Clin. Pharmacol. 1984; 18: 461-2. 
19 Cukier J, Ducassou J, Le Guillou M, Leriche A, Lobel B, Toubol J. Permixon versus placebo; resultats d'une étude multicentrique. C. R. Therapeut. Pharmacol. Clin. 1985; 4: 15-21.

20 Descotes JL, Rambeaud JJ, Deschaseaux P, Faure G. Placebocontrolled evaluation of the efficacy and tolerability of Permixon in benign prostatic hyperplasia after the exclusion of placebo responders. Clin. Drug Invest. 1995; 5: 291-7.

21 Emili E, Lo Cigno M, Petrone U. Risultati clinici su un nuovo farmaco nella terapia dell'ipertofia della prostata (Permixon). Urologia 1983; 50: 1042-8.

22 Gabric V, Miskic H. Behandlung des benignen prostataadenoms und der chronischen prostatatitis. Therapiewoche 1987; 37: 1775-88.

23 Lobelenz J. Extractum Sabal fructus bei benigner prostatahyperplasie (BPH). Klinische prufung im stadium I und II Therapeutikon 1992; 6: 34-7.

24 Mandressi S, Tarallo U, Maggioni A, Tombolini P, Rocco F, Quadraccia S. Terapia medica dell'adenoma prostatico: confronto della efficacia dell'estratto di Serenoa repens (Permixon ( ${ }^{\circledR}$ ) versus l'estratto di Pygeum Africanum e placebo. Valutazione in doppio cieco. Urologia 1983; 50: $752-8$

25 Mattei FM, Capone M, Acconcia A. Medikamentose therapie der benignen prostatahyperplasie mit einem extrakt der sagepalme. TW Urol. Nephrol. 1990; 2: 346-50.

26 Metzker H, Kieser M, Hölscher U. Wirksamkeit eines SabalUrtica-kombinationspräparats bei der behandlung der benignen prostatahyperplasie (BPH). Urologe B 1996; 36: 292-300.

27 Pannunzio E, D’Ascenzo R, Giardinetti F, Civili P, Persichelli E. Serenoa repens vs. gestonorone caproato nel trattamento dell'ipertofia prostatica benigna: Studio randomizzato. Urologia 1986; 53: 696-705.

28 Reece Smith H, Memon A, Smart CJ, Dewbury K. The value of permixon in benign prostatic hypertrophy. Br. J. Urol. 1986; 58: $36-40$.

29 Roveda S, Colombo P. Sperimentazione clinica controllata sulla bioequivalenza terapeutica e sulla tollerabilita dei prodotti a base di Serenoa repens in capsule da $160 \mathrm{mg}$ o capsule rettali da $640 \mathrm{mg}$. Arch. Med. Intern. 1994; 46: 61-75.

30 Sokeland J, Albrecht J. Kombination aus Sabal und Urticaestrakt vs. finasterid bei BPH (Stad. I bis II nach Alken); Vergleich der therapeutischen wirksamkeit in einer einjahrigen doppelblindstudie. Urologe A 1997; 36: 327-33.

31 Tasca A, Barulli M, Cavazzana A, Zattoni F, Artibani W, Pagano F. Trattamento della sintomatologia ostruttiva da adenoma prostatico con estratto di Serenoa repens. Studio clinico in doppio cieco vs. placebo. Minerva Urol. Nefrol. 1985; 37: 8791.

32 Boyle P, Gould AL, Roehrborn CG. Prostate volume predicts outcome of treatment of benign prostatic hyperplasia with finasteride: A meta-analysis of randomized clinical trials Urology 1996; 48: 398-405.

33 McConnell JD, Bruskewitz R, Walsh P, Andriole G, Lieber M, Holtgrewe L, et al. The effect of finasteride on the risk of acute urinary retention and the need for surgical treatment among men with benign prostatic hyperplasia. N. Engl. J. Med. 1998; 338: $557-63$.

34 Berges RR, Windeler J, Trampisch HJ, Senge TH. Randomised, placebo-controlled, double-blind clinical trial of $\beta$-sitosterol in patients with benign prostatic hyperplasia. Lancet 1995; 345: 1529-32.

35 Fischer A, Jurincic-Winkler CD, Klippel KF. Conservative treatment of benign prostatic hyperplasia with high-dosage $\beta$-sitosterol $(65 \mathrm{mg})$ : results of a placebo-controlled doubleblind study. Uroscop 1993; 1: 12-20.

36 Kadow C, Abrams PH. A double-blind trial of the effect of beta-sitosteryl glucoside (WA184) in the treatment of benign prostatic hyperplasia. Eur. Urol. 1986; 12: 187-9.

37 Klippel KF, Hiltl DM, Schipp B. A multicentric, placebo- controlled, double-blind clinical trial of $\beta$-sitosterol (phytosterol) for the treatment of benign prostatic hyperplasia. $\mathrm{Br}$. J. Urol. 1997; 80: 427-32.

38 AB Cernelle. Engleholm, Sweden.

39 Ruyan D, Cernitin American. Personal communication, 19 February 1999.

40 Buck AC. Phytotherapy for the prostate. Br. J. Urol. 1996; 78: 325-6.

41 Ito R, Ishii M, Yamashita S, et al. Antiprostatic hypertrophic action of Cernilton pollen-extract. Pharmacometrics 1986; 31 : $1-11$.

42 Kimura M, Kimura I, Nakase K, Sonobe T, Mori E. Micturition activity of pollen extract: contractile effects on bladder and inhibitory effects on urethral smooth muscle of mouse and pig. Planta Med. 1986; 2: 148-51.

43 Nakase S, Takenaka K, Hamanaka T, Kimura M. Effects of Cernilton pollen-extract on the urethral smooth muscle and diaphragmatic neuromuscular specimen. Folio Pharmacol. Jpn. 1988; 91: 385-92.

44 Becker H, Ebeling L. Konservative therapie der benignen prostata-hyperplasie (BPH) mit Cernilton ${ }^{\circledR} N$ : ergebnisse einer plazebokontrollierten doppleblindstudie. Urologe B 1988; 28 : 301-6.

45 Buck AC, Cox R, Rees RWM, Ebeling L, John A. Treatment of outflow tract obstruction due to benign prostatic hyperplasia with the pollen-extract, Cernilton: a double-blind, placebocontrolled study. Br. J. Urol. 1990; 66: 398-404.

46 Dutkiewicz S. Usefulness of Cernilton in the treatment of benign prostatic hyperplasia. Int. Urol. Nephrol. 1984; 28: 4953 .

47 Maekawa M, Kishimoto T, Yasumoto R, Wada S, Harada T, Ohara $\mathrm{T}$, et al. Clinical evaluation of Cernilton on benign prostatic hypertrophy: a multiple center double-blind study with Paraprost. Hinyo Kiyo 1981; 27: 317-26.

48 Andro MC, Riffaud JP. Pygeum africanum for the treatment of patients with benign prostatic hyperplasia: a review of 25 years of published experience. Curr. Ther. Res. 1995; 56: 796-817.

49 Barlet A, Albrecht J, Aubert A, Fischer M, Grof F, Grothuesmann HG, et al. Efficacy of Pygeum africanum extract in the medical therapy of urination disorders due to benign prostatic hyperplasia: evaluation of objective and subjective parameters. A placebo-controlled double-blind multicenter study. Wien. Klin. Wochenschr. 1990; 102: 667-73.

50 Barth H. Non hormonal treatment of benign prostatic hypertrophy. Clinical evaluation of the active extract of Pygeum africanum. Proceedings of Symposium on Benign Prostatic Hypertrophy, Paris 1981: 45-8.

51 Bassi P, Artibani W, De Luca V, Zattoni F, Lembo A. Standardized extract of Pygeum africanum in the treatment of benign prostatic hypertrophy. Controlled clinical study versus placebo. Minerva Urol. Nefrol. 1987; 39: 45-50.

52 Blitz M, Garbit JL, Masson JC, et al. Etude controlee de l'efficacite d'un traitemente medical sur des sujets consultant pour la premiere fois pour un adenome de la prostate. Lyon Mediterr. Med. 1985; 21: 11.

53 Bongi G. II Tadenan nella terapia dell'adenoma prostatico. Studio anatomo-clinico. Minerva Urol. 1972; 24: 124-38.

54 Donkervoort T, Sterling A, van Ness J, Donker PJ. A clinical and urodynamic study of Tadenan in the treatment of benign prostatic hypertrophy. Eur. Urol. 1977; 3: 218-25.

55 Dufour B, Choquenet C, Revol M, Faure G, Jorest R. Controlled study of the effects of Pygeum africanum extract on the functional symptoms of prostatic adenoma. Ann. Urol. 1984; 18: $193-5$.

56 Frasseto G, Bertoglio S, Mancuso S, Ervo R, Mereta F. Studio sull'efficacia e sulla tollerabilita del Tadenan 50 in pazienti affeti da ipertrofia prostatica. Prog. Med. 1986; 42: 49-53.

57 Gagliardi V, Apicella F, Pino P, Falchi M. Terapia medica dell'ipertrofia prostatica. Sperimentazione clinica controllata. Arch. Ital. Urol. Nefrol. Andrologia 1983; 55: 51-69. 
58 Giacobini S, von Heland M, de Natale G, Gentile V, Bracci U. Valutazione clinica e morfo-funzionale del trattamento a doppio cieco con placebo. Tadenan 50 e Tadenan 50 associato a Farlutal nei pazienti con ipertrofia prostatica benigna. Antologia Med. Ital. 1986; 6: 1-10.

59 Krzeski T, Kazon M, Borkowski A, Witeska A, Kuczera J. Combined extracts of Urtica dioica and Pygeum africanum in the treatment of benign prostatic hyperplasia: Double-blind comparison of two doses. Clin. Ther. 1993; 15: 1011-20.

60 Maver A. Medical treatment of fibroadenomatous hypertrophy of the prostate with a new plant substance. Minerva Med. 1972; 63: 2126-36.

61 Ranno S, Minaldi G, Viscusi G, Di Marco G, Consoli C. Efficacia e tollerabilita del trattamento dell' adenoma prostatico con Tadenan 50. Prog. Med. 1986; 42: 165-9.

62 Rigatti P, Zennaro F, Fraschini O, Oxilia A. L'impegio del
Tadenan nell'adenoma prostatico. Ricerca clinica controllata. Atti Accad. Med. Lomb. 1983; 38: 1-4.

63 Rizzo M, Tosto A, Paoletti MC, Raugei A, Favini P, Nicolucci A, et al. Terapia medica dell'adenoma della prostata: Valutazione clinica comparativa tra estratto di Pygeum africanum ad alte dosi e placebo. Farmacia Terapia 1985; 2: 105-10.

64 Englemann U, Boos G, Kres H. Therapie der benignen Prostatahyperplasie mit Bazoton liquidum. Urologe B 1996; 36: $287-91$.

65 Vontobel HP, Herzog R, Rutihauser G, Kres H. Results of a double-blind study on the effectiveness of ERU (extractum radicis Urticae) capsules in conservative treatment of benign prostatic hyperplasia. Urologe A 1985; 24: 49-51.

66 McKinney DE. Re: Saw palmetto for Benign Prostatic Hyperplasia. JAMA 1999; 281: 1699. 